\title{
Neue Prozesse verändern die Unternehmenskultur Positive Auswirkungen auf Arbeitszufriedenheit und Kosten
}

Das Universitätsklinikum ist der viertgrößte Arbeitgeber in Köln. 8600 Mitarbeiter aus verschiedenen Berufen arbeiten in den 58 Kliniken und Instituten auf dem Campusgelände. Sie versorgen jährlich gut 50000 Patienten stationär und mehr als 200000 Patienten ambulant. Der CMI ist von 1,4 in 2005 auf 1,6 in 2011 gestiegen. Das Klinikum erwirtschaftet ein positives Jahresergebnis. Dies, obwohl seit Jahren die Veränderungsrate des Landesbasisfallwertes nicht die Tarifsteigerungsraten bei den Personalkosten ausgleicht. Für 2011 errechnete sich daraus eine finanzielle Unterdeckung in Höhe von 7 Millionen Euro, die aufgefangen werden müssen. Günter Zwilling, seit 2008 Kaufmännischer Direktor der Uniklinik Köln, hat es sich daher zur Aufgabe gemacht, durch eine bessere Steuerung der innerbetrieblichen Abläufe die Kostenstruktur zu optimieren und damit die wirtschaftliche Zukunft des Klinikums langfristig zu sichern.

Wo setzen Sie an, um die innerbetrieblichen Abläufe zu optimieren? Günter Zwilling: Zu Beginn sprach ich mit den Direktoren der Kliniken und wollte wissen, welche Möglichkeiten es Günter Zwilling gibt, die Strukturen so

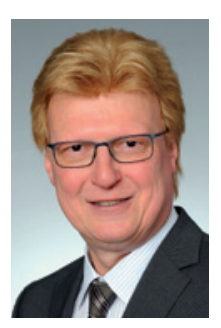

zu verbessern, dass Material eingespart und die Arbeit effizienter erbracht werden kann. Schnell führte uns dies zu der Frage, was die Verwaltung dazu beitragen und wie sie ihre Qualität als interner Dienstleister verbessern kann. Das heißt, wir müssen unsere Verwaltungsprozesse überdenken und effizienter gestalten.

\section{Wie sind Sie vorgegangen?}

Zwilling: Uns Vorstandsmitgliedern war es wichtig, das Wissen der Mitarbeiter aus der Verwaltung und den sekundären Dienstleistungen wie Technik, Küche, Reinigung einzubeziehen, denn sie wissen am besten, welche Abläufe verbesserungsbedürftig sind. Hierzu richteten wir 2 Workshops mit insgesamt 50 Beschäftigten aus. Als Ergebnis entstand eine Projektkarte mit verschiedenen Prozessvorhaben, beispielsweise im Beschaffungs- und Rechnungswesen, bei der Berichterstattung und im Umgang mit Verbesserungsvorschlägen. Mitarbeiter konnten sich als Prozessverantwortliche melden, wenn sie bereit waren, einen Prozess dauerhaft zu moderieren. Infolge bildeten sich etwa 30 Teams mit je 2 bis 4
Mitgliedern. Ein externes Beratungsunternehmen hat die Startphase begleitet. Zusätzlich haben wir in der Stabsabteilung Unternehmensentwicklung eine zentrale Projektmanagerin eingestellt.

\section{? „Rechnet“ sich die Mehrarbeit für Ihre Mitarbeiter?}

Zwilling: Eine zusätzliche Vergütung erhalten die Mitarbeiter nicht. Unser Ziel ist es, dass die Prozessverantwortlichen und ihre Teams am Ende des Tages merken, dass die neuen Prozesse ihre Arbeit einfacher machen. Früher gab es häufig Klagen über Doppelarbeit aufgrund unkoordinierter Arbeitsabläufe. Viele Verbesserungsvorschläge scheiterten. Die nötige Unterstützung auf Leitungsebene fehlte, was die Mitarbeiter demotiviert hat. Genau das wollen wir durch unser Verwaltungsprojekt verhindern.

\section{?elches Verfahren durchläuft ein neuer Verwaltungsprozess?}

Zwilling: In den Projektteams, die mit Mitarbeitern aus verschiedenen Abteilungen und Berufen besetzt sind, werden die neuen Prozessabläufe eigenständig erarbeitet und anschließend vom Vorstand genehmigt. In unsere Entscheidungen binden wird die Abteilungsleiter und die Prozessverantwortlichen ein.

\section{? Gibt es Akzeptanzprobleme?}

Zwilling: Ich nehme viel Interesse und Verständnis für Veränderungen wahr. Die Führungskräfte stehen hinter dem Projekt, zumal sie dabei von den operativen
Aufgaben entlastet sind. Die Zustimmung der Mitarbeiter ist ebenfalls hoch. Manchmal treten jedoch Zeitprobleme auf. Schwierig ist es momentan für die $\mathrm{Ar}$ beitsgruppen, Kennzahlen zu definieren, anhand derer die erfolgreiche Umsetzung eines Prozesses gemessen werden kann.

\section{? Welche Aufgaben und Befugnisse haben die Prozessverantwortlichen?} Zwilling: Sie leiten die Projektteams und sind verantwortlich für die kontinuierliche Prozessverbesserung. Sie stimmen die Prozesse in und zwischen den Abteilungen ab und achten darauf, dass sie funktionieren. Ich erhalte alle 4 Wochen einen Sachstandsbericht. Bei Störungen, Terminschwierigkeiten oder Unterstützungsbedarf können sich die Prozessverantwortlichen an ihren Vorgesetzen, an mich oder an den Leiter der zuständigen Abteilung wenden, wenn zum Beispiel die Kostenübernahme für eine Software geklärt werden muss. Der lange Weg über Abteilungshierarchien wird so durchbrochen. Oft vermittelt jedoch die Projektmanagerin, da es für die Prozessverantwortlichen noch ungewohnt ist, mich oder den Leiter einer anderen Abteilung direkt anzusprechen.

\section{Wie viele Prozesse sind bereits} eingeführt worden?

Zwilling: In der Verwaltung haben wir etwa 20 Prozesse eingeführt, 40 weitere sind in Arbeit. Patientenorientierte Prozesse wie Behandlungspfade in der Onkologie oder Pflegestandards sind unabhängig von dem zentralen Verwaltungsprojekt entstanden.

\section{Was verändert sich?}

Zwilling: Durch die Veränderung der Verwaltungsabläufe können unsere Mitarbeiter ihre Aufgaben schneller bearbeiten, da sie auf der Sachebene direkt zusammenarbeiten. Dadurch senken wir Kosten und verbessern unsere Liquidität. Zugleich verbessert sich die Qualität der Zusammenarbeit zwischen den Abteilungen. Die Mitarbeiter entwickeln untereinander ein besseres Verständnis für die 
Arbeit des anderen, sie lernen voneinander. Früher endeten Verbesserungsansätze oft an den Abteilungsgrenzen.

\section{? Können Sie die Kosteneffekte näher beschreiben?}

Zwilling: Wir haben zum Beispiel Skontoverluste, da wir Rechnungen aufgrund interner Verwaltungswege erst relativ spät begleichen. Um die Verwaltungswege zu verkürzen, soll die Ware künftig nicht mehr dezentral an die Institute und Kliniken, sondern an die Zentrale Warenannahme geliefert und dort elektronisch erfasst werden. Dadurch können wir auch auf das Gegenzeichnen der Rechnung durch den Besteller auf den Stationen verzichten. Zugleich stellen wir bis Ende des Jahres auf ein IT-gestütztes Anforderungssystem um. So können die Mitarbeiter den Bearbeitungsstand ihrer Bestellung jederzeit nachvollziehen und wenn es "hakt“, gezielt nachfragen. Dies soll dazu beitragen unsere hohen Lagerbestände zu reduzieren, denn bislang bestellen Mitarbeiter gern „auf Vorrat“ aus Sorge, die Ware könne nicht rechtzeitig eintreffen.

\section{? In welcher Höhe erwarten Sie Einsparungen?}

Zwilling: Wir gehen davon aus, dass wir in den nächsten 3 Jahren mindestens 3 Millionen Euro durch bessere Verwaltungsprozesse einsparen können. Das sind etwa $10 \%$ der direkten Verwaltungskosten. Beim Bürobedarf verzeichnen wir bereits Einsparungen in Höhe von 250000 Euro. Den Katalog der bestellbaren Büroartikel haben wir von 7500 auf ca. 1800 Artikel reduziert. Außerdem erhöhen wir unsere Liquidität erheblich, indem wir den Zeitraum der Rechnungsstellung für die Behandlungen durch verbesserte Kodierungsabläufe um 11 Tage verkürzen konnten. Pro Tag macht dies ca. 1,5 Millionen Euro aus.

\section{Ihr Fazit?}

Zwilling: Wir sind auf dem richtigen Weg und haben schon einiges bewegt. Unsere Mitarbeiter erkennen, dass durch die Arbeit an den Prozessen eine neue Dienstleistungskultur entsteht. Wir setzen auf Nachhaltigkeit und kontinuierliche Verbesserung, sodass das Klinikum seinen Anforderungen in personeller und wirtschaftlicher Hinsicht weiterhin gewachsen bleibt.

Das Interview führte Dr. Adelheid Weßling,

Düsseldorf 